\title{
Guidelines taugen nicht für Wirtschaftlichkeitsverfahren
}

Jürg Nadiga, Ernst Gähler ${ }^{b}$

a Präsident der Schweizerischen Gesellschaft für Medizinische Onkologie (SGMO)

b Vizepräsident der FMH, Verantwortlicher Ressort Tarife und Verträge; Verantwortlicher Rettungswesen; Verantwortlicher Ressort Paramedics
Der Bundesgerichtsentscheid vom 15. Dezember 2010 verbessert die Stellung des Arztes in Wirtschaftlichkeitsverfahren [1]. Er besagt, dass die statistische Methode ANOVA nur angewendet werden kann, wenn eine hinreichende Vergleichsbasis besteht. Ebenso hat die Vergleichsgruppe aus mindestens zehn Ärzten mit vergleichbarer Praxisstruktur zu bestehen und die Vergleichsperiode muss genügend lang sein. Darüber hinaus muss der Beklagte prüfen können, ob er mit einem homogenen Kollektiv verglichen wird, das eine ähnliche Praxisstruktur hat. Die zunehmende Spezialisierung der Ärzte erschwert

\section{Guidelines sind kein Kochbuch, und jeder Onkologe hat} das Recht und die Pflicht, in medizinisch begründeten Fällen zum Wohle des Patienten von den Richtlinien abzuweichen.

Korrespondenz:

Dr. med. Jürg Nadig MAE Facharzt für Medizinische Onkologie und Innere Medizin Mitglied der FMH Bannhaldenstrasse 7 CH-8180 Bülach juerg.nadig(at)hin.ch es, diese Voraussetzungen für das statistische Verfahren zu erfüllen. Deshalb kommt bei Wirtschaftlichkeitsverfahren gegen Spezialisten vermehrt die analytische Methode zur Anwendung. In einem laufenden Gerichtsverfahren versucht santésuisse für diese Fälle ein für sie vorteilhaftes Präjudiz zu schaffen. santésuisse fordert, dass der Experte die zu beurteilenden Fälle mit den Guidelines einer von ihr bestimmten Organisation zu vergleichen habe; Behandlungen, die über die Guidelines hinausgehen, seien demnach unwirtschaftlich.

Gemäss santésuisse liege es im Interesse der Patienten und Prämienzahler, dass diese Guidelines genau dann herangezogen würden, wenn die durchschnittlichen Behandlungskosten eines zu überprüfenden Arztes ein gewisses Niveau überstiegen. Dabei könne festgestellt werden, ob die deutlich höheren Behandlungskosten mit dem Gebot der Wirtschaftlichkeit im Einklang stünden und die Qualitätsstandards eingehalten seien.

Zweifelsohne liegt es im Interesse des Patienten, höhere Behandlungskosten mittels Guidelines zu rechtfertigen. Falsch ist aber die Annahme von santésuisse, Guidelines würden sich auch eignen, Mehrkosten als unwirtschaftlich zu qualifizieren, die durch eine von den Guidelines abweichende Behandlung entstehen. Guidelines definieren einen Minimalstandard für die Behandlungsqualität. Zudem werden in
Les guides de pratique ne sont pas utiles pour déterminer le caractère économique des prestations

Les décisions récentes du Tribunal fédéral renforcent la position des médecins dans les procédures portant sur le caractère économique des prestations. La méthode statistique ANOVA ne doit être utilisée que s'il existe une base de comparaison suffisante. En raison de la spécialisation croissante des médecins, on aura de plus en plus souvent recours à une méthode analytique dans les procédures visant à évaluer le caractère économique des prestations fournies par des spécialistes. Dans une procédure judiciaire en cours, santésuisse tente de créer un précédent qui lui serait favorable dans ces cas-là. Selon santésuisse, les traitements allant au-delà des normes minimales des guides de pratique ne répondraient pas aux critères d'économicité. Or les études utilisées pour I'élaboration des guides de pratique ne reflètent pas assez bien la pratique quotidienne des médecins. De par leur qualité de «patient idéal», les personnes participant à ces études ne représentent qu'une minorité. Le traitement de patients polymorbides (moyens) est souvent plus complexe et s'avère plus coûteux à cause des diverses maladies dont ils souffrent. Si les tribunaux et les politiques se ralliaient à la position de santésuisse, les normes minimales dans l'assurance de base deviendraient le maximum de ce que la médecine est autorisée à apporter. Les guides de pratique médicaux ne représenteraient ainsi plus un soutien thérapeutique pour le médecin, mais un instrument de rationalisation implicite des assureurs.

den Studien, die den Guidelines zugrunde liegen, idealtypische Patienten behandelt. Diese unterscheiden sich in den Behandlungskosten logischerweise von 


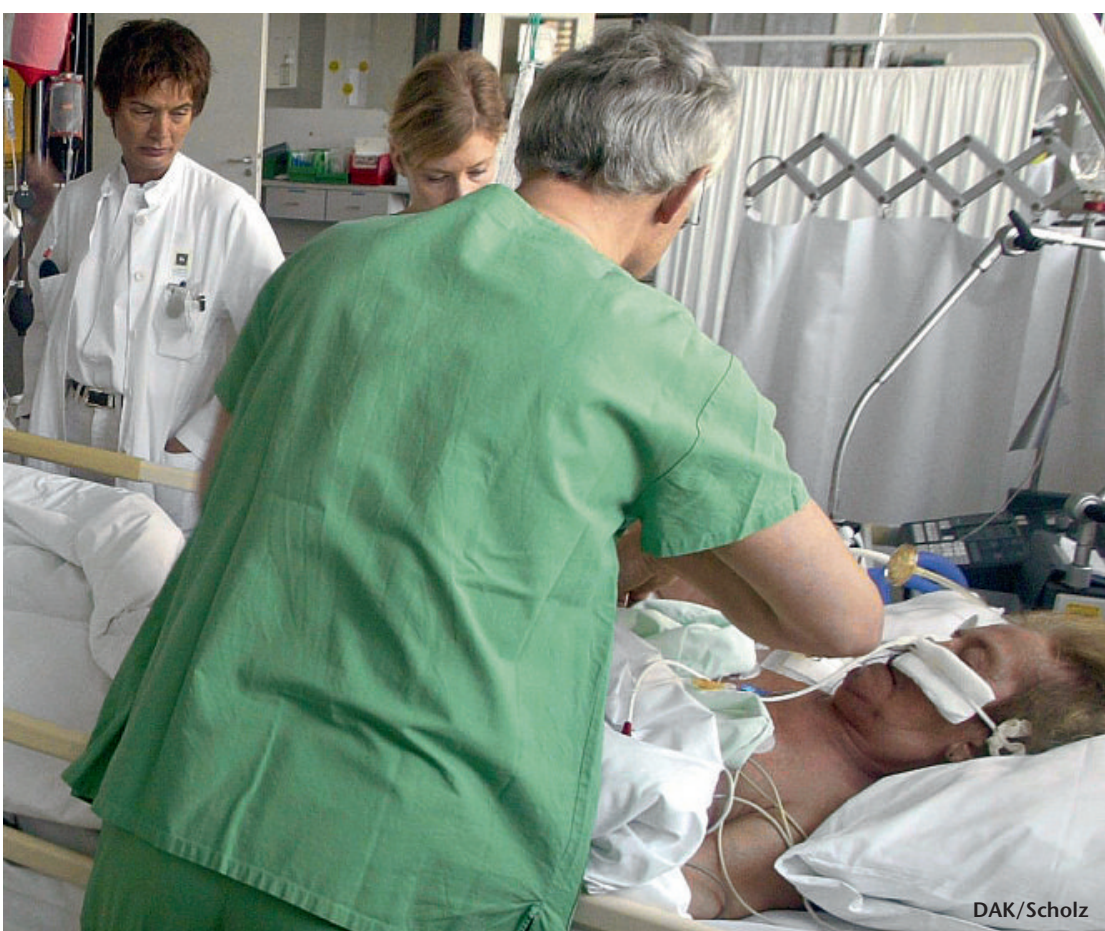

In der täglichen Praxis entsprechen die Kranken nicht den idealtypischen Patienten der Studien. Guidelines verpflichten den Arzt auch dazu, im Interesse des Patienten von ihnen abzuweichen.
Fachgesellschaft fest, die Festlegung dieser minimalen Eckdaten diene der Qualität der ärztlichen Tätigkeit. Sie seien aber nicht dazu da, den behandelnden Arzt einzuschränken. Für die Fachgesellschaft war es bereits damals wichtig, dass ihre Mitglieder gegenüber Krebspatienten auf eine qualitativ hochstehende Betreuung und Behandlung hinweisen konnten. Die Guidelines, auch wenn sie veröffentlicht wurden, waren primär für die Mitglieder der Fachgesellschaft abgefasst. Die SGMO war bereits damals der Meinung, Guidelines müssten vom Medizinischen Onkologen interpretiert und patientengerecht angewendet werden. Sie seien jedoch kein Kochbuch, und jeder Onkologe habe das Recht und die Pflicht, in medizinisch begründeten Fällen zum Wohle des Patienten von den Richtlinien abzuweichen.

In gleicher Weise äussern sich auch heute internationale Guidelines. So schreibt das National Comprehensive Cancer Network (NCCN), die Richtlinien fassten die Evidenz zusammen und stellten einen Konsens der Autoren bezüglich ihrer Sicht der zurzeit anerkannten Behandlungsmöglichkeiten dar. Von jedem Kliniker, der diese Empfehlungen anwende oder konsultiere, werde aber eine unabhängige medizinische Beurteilung der individuellen klinischen Situation des Patienten erwartet, bevor er dessen Pflege oder Therapie festlege. Gemäss Physician Data Query (PDQ) des National Cancer Institute seien Guidelines jedoch keine Empfehlungen für Entscheide im Gesundheitswesen.

Gleiches gilt für die ESMO-Guidelines, auf die sich santésuisse beruft: Sie stellen lediglich einen Konsens unter den (wenigen) Autoren dar, welche die Guidelines ausgearbeitet haben. Diese Experten haben kein nationales Mandat und die inhaltliche Qualität der Guidelines ist sehr unterschiedlich.

Ihre Empfehlungen basieren auf Studien, die nur für ein wohldefiniertes Patientenkollektiv gelten. Die Patienten in der Sprechstunde leiden jedoch oft an verschiedenen Zusatzerkrankungen. Sie haben einen unterschiedlichen kulturellen und sozialen Hintergrund und entsprechen in der Regel nicht dem idealtypischen Studienpatienten. Die ESMO-Guidelines umschreiben einen minimalen Behandlungsstandard, der längerfristig allen Einwohnern der EU zukommen sollte. Diese Richtlinien als Positivliste für eine maximal zulässige Medizin zu definieren und Abweichungen davon als unwirtschaftlich zu qualifizieren, wäre fatal: Aus einem anzustrebenden Minimalstandard würde eine im Voraus definierte Maximalmedizin.

Ein legaler Stellenwert könnte den Guidelines höchstens zur Beurteilung einer Unterversorgung zukommen: Ein Onkologe hat gegenüber den Patienten oder der Haftpflichtversicherung zu begründen, weshalb er diesen Minimalstandard nicht eingehalten hat. Gegenüber den Kostenträgern eignen sich Guidelines, um unberechtigte Vorwürfe bezüglich Überarztung abzuwehren. Deshalb können Behandlungen, Medizinische Onkologie (SGMO) sieben Guidelines
ausgearbeitet und in einem internen Vernehmlassungsprozess angepasst [2]. Bereits damals hielt die

wohl in ihrer Ausführlichkeit als auch in den emp Medikamenten. Die diagnostischen weise auch die lokalen Gegebenheiten. Die Schweize rische Gesellschaft für Medizinische Onkologie (SGMO) sah bis heute keine Notwendigkeit, eine dieser Richtlinien für die Onkologen in der Schweiz als verbindlich zu erklären.

1998 hatte die Schweizerische Gesellschaft für Medizinische Onkologie (SGMO) sieben Guidelines
ausgearbeitet und in einem internen Vernehmlas-

Patienten mit Mehrfacherkrankungen. Trotz Guidetient verlangt eine optimale Behandlung und der Vericherte will möglichst tiefe Krankenkassenprämien.
In der Onkologie bestehen neben den Guidelines der European Society for Medical Oncology (ESMO) noch andere Sammlungen von Empfehlungen
(NCCN, British Columbia Cancer Agency, ASCO, PDQ NCI, Onkopedia). Diese unterscheiden sich so-

\section{Behandlungen, die nicht in den Guidelines aufgeführt sind, können nicht automatisch als unwirtschaftlich gelten.}


die nicht in den Guidelines aufgeführt sind, nicht automatisch als unwirtschaftlich gelten. Im Gegenteil: Die Behandlung von Patienten mit verschiedenen $\mathrm{Zu}$ satzerkrankungen dürfte sogar teurer sein als die Therapie von Studienpatienten ohne Zusatzerkrankungen. Was aber kehren die Kostenträger vor, um zu ver-

\section{Ein legaler Stellenwert könnte den Guidelines}

\section{höchstens zur Beurteilung einer Unterversorgung}

\section{zukommen.}

hindern, dass die Ärzte die Patienten aus Angst vor Wirtschaftlichkeitsverfahren unter dem Minimalstandard behandeln? Es wäre bedenklich, wenn die Versicherer Patienten mit Zusatzerkrankungen benachteiligten, um die Prämien möglichst tief zu halten.

Bei nichtkurativen Behandlungen sind viele Behandlungsoptionen offen, die nicht im Detail in den Guidelines beschrieben werden. Deshalb ist der Nutzen individuell zu beurteilen und mit dem Patienten abzusprechen. Zudem muss der behandelnde Onkologe davon ausgehen können, dass die WZW-Kriterien erfüllt sind, wenn er Medikamente aus der Spezialitätenliste verabreicht, die für diese Indikation registriert oder vom Vertrauensarzt für einen Off-Labeluse bewilligt sind, auch wenn sie in den ESMO-Guidelines (noch) nicht aufgeführt sind.

Guidelines im analytischen Wirtschaftlichkeitsverfahren zu verwenden verletzt deren Geist in dreifacher Hinsicht:

1. Dem Sinn der Einzelfallbeurteilung wird nicht Rechnung getragen, weil man ja gerade nicht den einzelnen Fall im medizinischen Kontext beurteilt. Es wird lediglich ein Behandlungsstandard schematisch auf den Einzelfall abgebildet. Die Guideline-Konformität würde zum Wirtschaft- lichkeitskriterium einer Behandlung umfunktioniert, statt als Qualitätsindikator gegen eine Unterversorgung $\mathrm{zu}$ dienen.

2. Die in den Guidelines verwendeten Studien bilden die tägliche Praxis ungenügend ab. Studienpatienten stellen als «ideale» Patienten eine Minderheit dar. Patienten mit Mehrfacherkrankungen werden in der Regel von Studien ausgeschlossen. In der täglichen Praxis entsprechen die Kranken nicht den idealtypischen Patienten der Studien. Die Behandlung von mehrfachkranken (Durchschnitts-)Patienten ist aber gerade wegen den $\mathrm{Zu}$ satzerkrankungen aufwendiger und damit kostenintensiver. Daraus den Schluss zu ziehen, diese Behandlungen seien unwirtschaftlich, würde die Mehrzahl der Krebskranken von einer modernen Behandlung ausschliessen.

3. Guidelines stellen nur einen Minimalkonsens unter den Autoren dar. Sie bieten dem Arzt Behandlungshilfen, indem sie die Fakten aus den vorliegenden Studien zusammenfassen. Guidelines verpflichten den behandelnden Arzt aber explizit, im Interesse des Patienten in einzelnen Situationen davon abzuweichen.

Übernähmen Gerichte und Politik die Sicht von santesuisse, würde in der Grundversicherung der Minimalstandard zur maximal zulässigen Medizin umfunktioniert. Guidelines im Gesundheitswesen wären damit nicht mehr Behandlungshilfen für den Arzt, sondern implizite Rationierungsinstrumente der Versicherer. Das können und sollen Guidelines aber explizit nicht sein.

\section{Literatur}

1 Urteil des Bundesgerichtes vom 15. Dezember 2010 (Verfahren 9C_968/2009).

21 SGIO Quality Assessment Group. Guidelines. Schweiz Ärztezeitung. 1999;80:137-45. 